\title{
kGCN: a graph-based deep learning framework for chemical structures
}

$\operatorname{AUTHOR}(S)$ :

Kojima, Ryosuke; Ishida, Shoichi; Ohta, Masateru; Iwata, Hiroaki; Honma, Teruki; Okuno, Yasushi

\section{CITATION:}

Kojima, Ryosuke ...[et al]. kGCN: a graph-based deep learning framework for chemical structures. Journal of Cheminformatics 2020, 12: 32.

\section{ISSUE DATE:}

2020

URL:

http://hdl.handle.net/2433/261684

\section{RIGHT:}

(C) The Author(s) 2020. This article is licensed under a Creative Commons Attribution 4.0 International License, which permits use, sharing, adaptation, distribution and reproduction in any medium or format, as long as you give appropriate credit to the original author(s) and the source, provide a link to the Creative Commons licence, and indicate if changes were made. The images or other third party material in this article are included in the article's Creative Commons licence, unless indicated otherwise in a credit line to the material. If material is not included in the article's Creative Commons licence and your intended use is not permitted by statutory regulation or exceeds the permitted use, you will need to obtain permission directly from the copyright holder. To view a copy of this licence, visit http://creativecommons.org/licenses/by/4.0/. The Creative Commons Public Domain ... 


\title{
kGCN: a graph-based deep learning framework for chemical structures
}

\author{
Ryosuke Kojima ${ }^{1 *} \mathbb{D}$, Shoichi Ishida², Masateru Ohta ${ }^{3}$, Hiroaki Iwata', Teruki Honma ${ }^{3,4}$ and Yasushi Okuno ${ }^{1,3}$
}

\begin{abstract}
Deep learning is developing as an important technology to perform various tasks in cheminformatics. In particular, graph convolutional neural networks (GCNs) have been reported to perform well in many types of prediction tasks related to molecules. Although GCN exhibits considerable potential in various applications, appropriate utilization of this resource for obtaining reasonable and reliable prediction results requires thorough understanding of GCN and programming. To leverage the power of GCN to benefit various users from chemists to cheminformaticians, an open-source GCN tool, kGCN, is introduced. To support the users with various levels of programming skills, kGCN includes three interfaces: a graphical user interface (GUI) employing KNIME for users with limited programming skills such as chemists, as well as command-line and Python library interfaces for users with advanced programming skills such as cheminformaticians. To support the three steps required for building a prediction model, i.e., pre-processing, model tuning, and interpretation of results, kGCN includes functions of typical pre-processing, Bayesian optimization for automatic model tuning, and visualization of the atomic contribution to prediction for interpretation of results. kGCN supports three types of approaches, single-task, multi-task, and multi-modal predictions. The prediction of compound-protein interaction for four matrixmetalloproteases, MMP-3, $-9,-12$ and -13 , in the inhibition assays is performed as a representative case study using kGCN. Additionally, kGCN provides the visualization of atomic contributions to the prediction. Such visualization is useful for the validation of the prediction models and the design of molecules based on the prediction model, realizing "explainable Al" for understanding the factors affecting Al prediction. kGCN is available at https://github.com/clinfo.
\end{abstract}

Keywords: Graph convolutional network, kGCN, Graph neural network, Open source software, KNIME

\section{Introduction}

Deep learning is emerging as an important technology to perform various tasks in cheminformatics [1-3]. With the recent development of artificial intelligence (AI) and deep learning, the application of deep learning approaches has been practically demonstrated for various predictions such as virtual screening [4], quantitative structure-activity relationship (QSAR) studies [5], and ADMET (absorption, distribution, metabolism elimination, and toxicology) prediction $[6,7]$. In particular, with

*Correspondence: kojima.ryosuke.8e@kyoto-u.ac.jp

${ }^{1}$ Graduate School of Medicine, Kyoto University, Shogoin-kawaharacho, Sakyo-ku, Kyoto 606-8507, Japan

Full list of author information is available at the end of the article the democratization of AI, it is expected that these prediction tools should be readily used by the non-experts. The accessibility of deep learning to non-experts is an important issue in the field of cheminformatics. For example, as deep learning can be applied to a wide range of research areas in drug discovery such as ADMET predictions for lead optimization and virtual screening for lead identification, the chemists should be able to solve these research problems by using the latest technologies and analyze the results, availing the benefits of deep learning. However, as chemists are typically not proficient in deep learning, the development of easy-to-use, multi-functional deep learning software is necessary.

In the predictions based on molecular structures, graph neural networks (GNNs), where a chemical

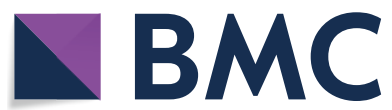

(c) The Author(s) 2020. This article is licensed under a Creative Commons Attribution 4.0 International License, which permits use, sharing, adaptation, distribution and reproduction in any medium or format, as long as you give appropriate credit to the original author(s) and the source, provide a link to the Creative Commons licence, and indicate if changes were made. The images or other third party material in this article are included in the article's Creative Commons licence, unless indicated otherwise in a credit line to the material. If material is not included in the article's Creative Commons licence and your intended use is not permitted by statutory regulation or exceeds the permitted use, you will need to obtain permission directly from the copyright holder. To view a copy of this licence, visit http://creativeco mmons.org/licenses/by/4.0/. The Creative Commons Public Domain Dedication waiver (http://creativecommons.org/publicdomain/ zero/1.0/) applies to the data made available in this article, unless otherwise stated in a credit line to the data. 


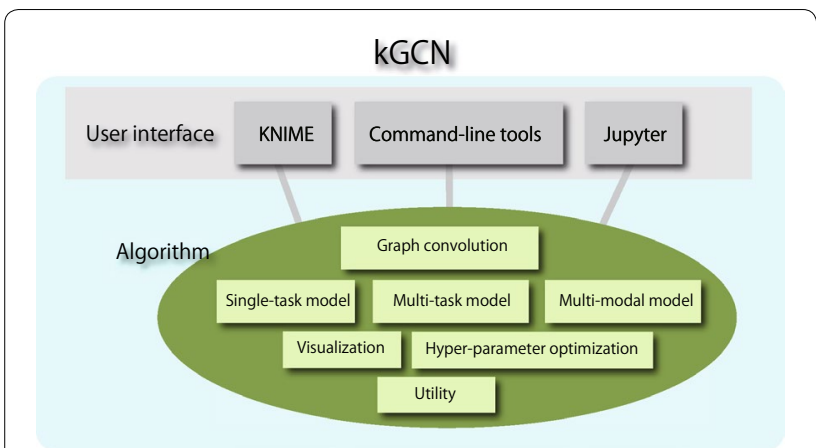

Fig. 1 Architecture of $k G C N$

structure is represented as a graph, have been reported to perform well $[8,9]$. In particular, graph convolutional networks (GCNs), a type of GNN, exhibited excellent performances in many applications [10, 11]. Despite these results, an appropriate application of GCN to realworld research problems requires practical programming skills and comprehensive understanding of deep learning and GCN.

To address this issue, a new open-source software, $\mathrm{kGCN}^{1}$. is introduced for various users to employ deep learning including GCNs. kGCN is developed for the following functions:

- Providing interfaces for the various levels of users including users with limited programming skills

- Handling different types of data for cheminformatics tasks

- Easy, intuitive, and convincing interpretation of results

- Hyper-parameter optimization

As mentioned, one function of $\mathrm{kGCN}$ is to afford interfaces to assist various users such as chemists, cheminformaticians, and data scientists. Considering the expertise levels of these users, a software should provide multiple interfaces suitable for each user. To satisfy these requirements, kGCN provides three types of user interfaces. Figure 1 shows the architecture of the kGCN system. The kGCN system supports both GUI-based and commandline interfaces. To intuitively access a machine-learning procedure, the kGCN system provides a GUI interface on the GUI platform, KNIME (Konstanz Information Miner) [12]. The command-line interface supports typical machine-learning procedures such as training, evaluation, and cross-validation. Additionally, the $\mathrm{kGCN}$

${ }^{1}$ Kyoto-university graph convolutional network framework. modules can be used as a Python library to allow flexibility and processing through programming languages.

The second function is to support different types of data. In cheminformatics, various types of data including chemical structures represented by graphs shoulded be considered. For example, the protein sequence data is often represented as a symbol sequence or vector descriptor. In deep learning, various architectures for neural networks have been proposed [13]. The simplest GCN is based on the single-graph-input singlelabel-output architecture. The kGCN system supports 1) multi-input (multi-modal GCN) and 2) multi-output (multi-task GCN) architectures. A multi-modal GCN is a neural network that can accept multiple modalities of inputs $[14,15]$. kGCN can accommodate a neural network with two inputs: chemical structure as a graph and a protein sequence as a series of characters. This type of neural network can be used to predict interactions between the compounds and proteins for virtual screening and/or drug-repurposing $[4,16]$. However, multiple related tasks are needed to be simultaneously handled in cheminformatics [17], for example, tasks to predict multiple different properties of a compound. To tackle these, a multi-task neural network is applied, which affords better results than those for an individual prediction [18, 19].

The third function is the interpretation and understanding of the cause of prediction results via deep learning by visualizing contributions of input data to the prediction. This process is important because the validity of the prediction model can be examined through a visual inspection of the good and bad features. The refinement or re-construction of the prediction model can be performed if the causes of prediction do not appear to be reasonable or are contrary to common sense. Notably, designing new molecules with improved properties is possible if the reasons for good and/or bad predictions are identified by visualization. In recent years, several methods to calculate the different contributions to the prediction results of deep learning have been proposed $[20,21]$. The kGCN system uses the integrated gradient method [22], which can be applied to any type of neural network architectures including multi-task and multimodal neural networks.

The last function is hyper-parameter optimization. In analysis using deep neural networks, hyper-parameters of deep learning such as the number of network layers, number of layer nodes, learning rate, and batch size should be appropriately set. However, setting these parameters is not easy for users without deep learning knowledge and experience. To assist such users and automatically determine the optimal hyper-parameters, 
the kGCN system employs Bayesian optimization and metaheuristics for hyper-parameter optimization [23].

In addition to this information, the kGCN system also provides tools for improving the usability. The kGCN back-end implementation uses Tensorflow [24] and supports GPUs (graphics processing units). To setup the execution conditions, kGCN-installed Docker images are also provided ${ }^{2}$. Additional unique tools to enhance the usability are provided for each interface. These will be described in the Implementation section.

Similar types of software have been reported in prior studies, e.g., DeepChem [25], Chainer chemistry [26], and OpenChem [27]. DeepChem is a Python library for neural networks, including GCNs. A notable feature of DeepChem is to support various machine learning methods as well as deep learning methods. Because deep learning usually requires large amounts of data, this feature can help users handle relatively small amounts of data. Chainer chemistry provides GCNs as an extended Python library of Chainer [28]. Both libraries can be used with Python and were developed for professional programmers of machine learning and Python. Although OpenChem supports both command-line and Python interfaces, good programming skills are still required to use OpenChem. The kGCN system is a framework containing the GUI, command-line, and Python interfaces. The GUI interface of $\mathrm{kGCN}$ is expected to engage users with limited programming skills in GCN and deep learning. To our knowledge, kGCN is the first open-source and multi-functional GCN software to support all three interfaces.

\section{Implementation}

Before describing the details of the kGCN system, basic implementation techniques for the graph representation of molecules and graph convolution are discussed.

\section{Graph representation of molecules for GCN}

This section first describes the formalization of a molecule to apply the GCNs. A molecule is formalized as a tuple $\mathcal{M} \equiv(V, E, F)$, where $V$ is a set of nodes. A node represents an atom in a molecule. A node has features $\mathbf{f}_{i} \in F(i \in V)$, and $F$ is a set of feature vectors representing the atom properties such as atom type, formal charge, and hybridization. These features should be appropriately designed by users. $E$ is a set of edges, and an edge $e \in E$ represents a bond between the atoms, i.e., $e \in V \times V \times T$, where $T$ is a set of bond types. An adjacency matrix $\mathbf{A}^{(t)}$ is used, which is defined as follows:

$$
\left(\mathbf{A}^{(t)}\right)_{i, j}=\left\{\begin{array}{ll}
1 & \left(v_{i}, v_{j}, t\right) \in E \\
0 & \left(v_{i}, v_{j}, t\right) \notin E
\end{array},\right.
$$

where $(\cdot)_{i, j}$ represents the $j$-th element of $i$-th row. Similarly, the feature matrix is defined as:

$$
(\mathbf{F})_{j, k}=\left(\mathbf{f}_{j}\right)_{k}
$$

where $(\cdot)_{k}$ represents the $k$-th element of a vector.

Using this matrix, a molecule is represented by $\mathcal{M}^{\prime}=(\mathbf{A}, \mathbf{F})$, where $\mathbf{A}=\left\{\mathbf{A}^{(t)} \mid t \in T\right\}$. The framework in the present system uses RDKit [29] to create adjacency and feature matrices and employs $\mathcal{M}^{\prime}$ as the input for GCN.

\section{Graph convolutional network}

kGCN supports GCNs in addition to the standard feedforward neural networks. Therefore, GCNs for molecules are described first. Graph convolution layer, graph dense layer, and graph gather layer are defined as described below.

\section{Graph convolution layer}

The graph convolution is calculated from the input $\mathbf{X}^{(\ell)}$ of the $\ell$-th layer as follows:

$$
\mathbf{X}^{(\ell+1)}=\sigma\left(\sum_{t} \tilde{\mathbf{A}}^{(t)} \mathbf{X}^{(\ell)} \mathbf{W}_{t}^{(\ell)}\right),
$$

where $\mathbf{X}^{(\ell)}$ is the $N \times D^{(\ell)}$ matrix and $\mathbf{W}_{t}^{(\ell)}$ is the parameter matrix $\left(D^{(\ell)} \times D^{(\ell+1)}\right)$ for a bond type $t, \sigma$ is the activation function, and $\tilde{\mathbf{A}}^{(t)}$ is the normalized adjacency matrix $(N \times N)$. This normalization and implementation of the layers follows Kipf's model [30] as a default. There are various choices for implementing the setting of graph convolution layers. In the $\mathrm{kGCN}$ system, the operation of the first layer input can be easily switched by changing the initial setting file for building the model.

The GCN is based on this graph convolution operation. The input of the first layer $\mathbf{X}^{(1)}$ often corresponds to the feature matrix, $\mathbf{F}$

\section{Graph dense layer}

$\mathbf{X}^{(\ell)}$ is an input for graph dense layer. $\mathbf{X}^{(\ell+1)}$ is calculated as follows:

$$
\mathbf{X}^{\ell+1}=\mathbf{X}^{(\ell)} \mathbf{W}^{(\ell)},
$$

where $\mathbf{X}^{(\ell)}$ is an $N \times D^{(\ell)}$ matrix and $\mathbf{W}^{(\ell)}$ is a parameter $\operatorname{matrix}\left(D^{(\ell)} \times D^{(\ell+1)}\right)$.

\section{Graph gather layer}

This layer converts a graph into a vector [31], i.e., the input $\mathbf{X}^{(\ell)}$ is an $N \times D^{(\ell)}$ matrix and $\mathbf{X}^{(\ell)}$, i.e., 


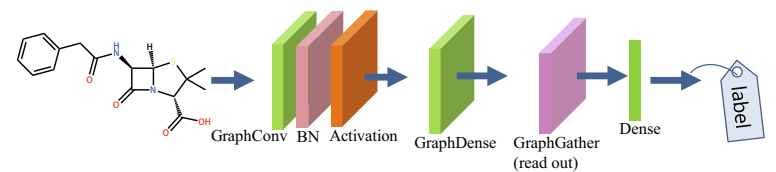

Fig. 2 Graph convolutional network for a prediction task with a compound input

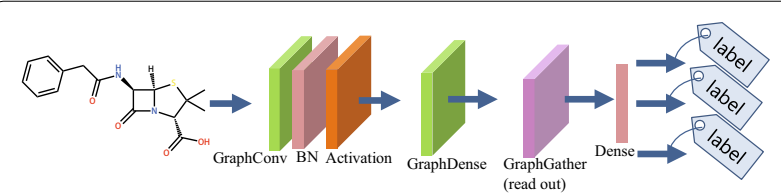

Fig. 3 Multi-task graph convolutional network with a compound input

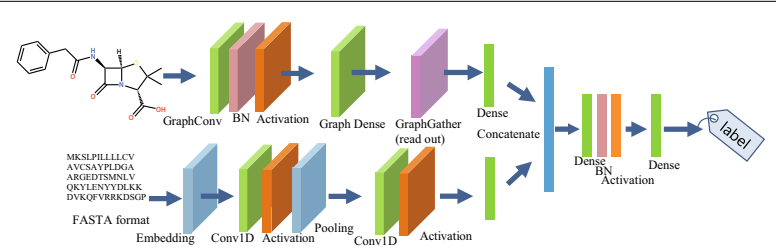

Fig. 4 Multi-modal graph convolutional network with compound and sequence inputs

$$
\left(\mathbf{X}^{(\ell+1)}\right)_{j}=\sum_{j}\left(\mathbf{X}^{(\ell)}\right)_{i j},
$$

where $(\cdot)_{i}$ represents an $i$-th element of a vector. This operation converts a matrix into a vector.

Figure 2 shows an example of GCN for a prediction task. The GCN model is a neural network consisting of a graph convolutional layer (GraphConv) with batch normalization (BN) [32] and rectified linear unit (ReLU) activation, graph dense layer with the ReLU activation, graph gather layer, and dense layer with the softmax activation. By assigning the label that is suitable for each task to the compounds, this model can be applied to many types of tasks, e.g., ADMET prediction based on the chemical structures.

Figure 3 shows an example of a multi-task GCN for a prediction task. The only difference is that multiple labels are predicted as an output. In this type of neural networks, multiple labels associated with a molecule such as several types of ADMET properties can be predicted simultaneously. It is well-known that multi-task prediction affords more improvement in the performance compared to that of individual single-task prediction [33].
Figure 4 shows an example of a multi-modal neural network employing a graph representing a compound and sequence of a protein. In addition to the information derived from the molecular structure, information from other modalities can also be used for the input. An example of the prediction of activity using compound and protein related information is described in detail in the Experiment section.

The kGCN system supports operations described above and some other additional operations to build a neural network. These operations are implemented using TensorFlow [34] and are compatible with Keras [35], allowing the users to construct neural networks such as convolutional neural networks and recurrent neural networks [13] with Keras operations.

These neural networks include hyper-parameters such as the number of layers in a model and number of dimensions for each layer. To determine these hyper-parameters, the kGCN system includes Bayesian optimization.

\section{Visualization of graph convolutional network}

To confirm the features of the molecules that influence prediction result, a visualization system using the integrated gradient (IG) method [22] is developed. After the construction of the prediction model, the visualization of the atom importance in the molecular structure, based on the IG value $\mathcal{I}(x)$ derived from the prediction model, is possible.

IG value $\mathcal{I}(x)$ is defined as follows:

$$
\mathcal{I}(x)=\frac{x}{M} \sum_{k=1}^{M} \nabla S\left(\frac{k}{M} x\right),
$$

where $x$ is the input of an atom of a molecule, $M$ is the number of divisions of the input, $S(x)$ is the prediction score, i.e., the neural network output with input $x$, and $\nabla S(x)$ is the gradient of $S(x)$ related to input $x$. In the default setting, $M$ is set to 100 . The atom importance is defined as the sum of the IG values of features in each atom. The calculation of the atom importance is performed on compound-by-compound basis.

The evaluation of the visualization results depends on each case. Although methods for the visualization of deep learning results are still developing, their effectiveness in solving common problems has not been reported; however, a quantitative evaluation of the IG values related to the molecules was previously reported for the prediction of a reaction [36].

\section{Hyper-parameter optimization}

To optimize the neural network models, hyper-parameters such as the number of graph convolution layers, the number of dense layers, dropout rate, and learning rate 
should be determined. As it is difficult to manually determine all these hyper-parameters, kGCN allows automatic hyper-parameter optimization with Gaussian-processbased Bayesian optimization using a Python library, GPyOpt [37].

\section{Interfaces}

This section describes three interfaces in the kGCN system.

\section{Command-line interface}

The kGCN system provides the command-line interface suitable for batch execution. Data processing is designed according to the aim, but there is a standard process common to many data processing designs, e.g., a series of processes for cross-validation. The kGCN commands include these common processes, i.e., the $\mathrm{kGCN}$ system allows preprocessing, learning, prediction, cross-validation, and Bayesian optimization using the following commands:

kgcn-chem command allows preprocessing of molecule data, e.g., structure-data file (SDF) and SMILES.

kgcn command allows batch execution related to prediction tasks: supervised training, prediction, cross-validation, and visualization.

kgcn-opt command allows batch execution related to hyper-parameter optimization.

These commands can be used with Linux commands and enable users to construct automatic scripts, e.g., Bash scripts. Because such batch execution is suitable for large-scale experiments using workstation and reproducible experiments, this interface is useful for the evaluation of neural network models.

\section{KNIME interface}

The kGCN system supports KNIME modules as a GUI. KNIME is a platform to prepare the workflow, which consists of KNIME nodes for data processing, and is particularly useful in the field of data science. The kGCN KNIME nodes described below are useful for the execution of various $\mathrm{kGCN}$ functions in combination with existing KNIME nodes. The command-line interface allows batch execution, whereas the KNIME interface is suitable for early steps in the machine learning process such as prototyping and data preparation.

To train and evaluate the model, $\mathrm{kGCN}$ provides the following two nodes.

GCNLearner

GCNPredictor

Using the kGCN nodes mentioned above, Fig. 5 shows an example of the workflow. This data flow can be separated into that before and after GCNLearner. The former part is for data preparation, for which kGCN includes the following KNIME nodes:

CSVLabelExtractor

SDFReader

\section{GraphExtractor}

AtomFeatureExtractor

GCNDatasetBuilder

\section{GCNDatasetSplitter}

The test dataset is used for the evaluation and interpretation of results. kGCN also provides the modules to display the output of the results.

GCNScore

GCNScoreViewer

GCNVisualizer

GCNGraphViewer

Another example of the workflow is shown in Fig. 6 , which includes an example of multi-modal neural networks. To design multi-modal neural networks, the kGCN system provides the following modules:

AdditionalModalityPreprocessor reads the data of another modality from a given file. 


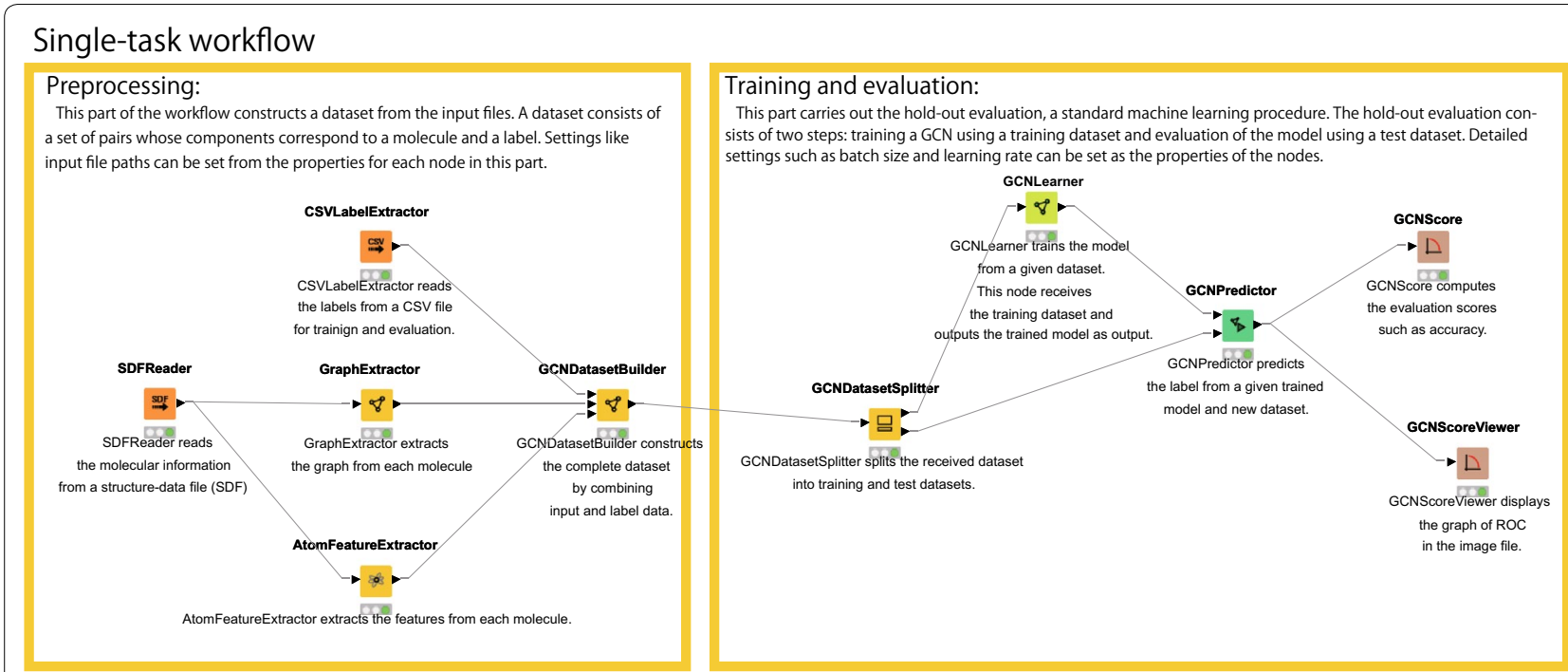

Multi-task workflow

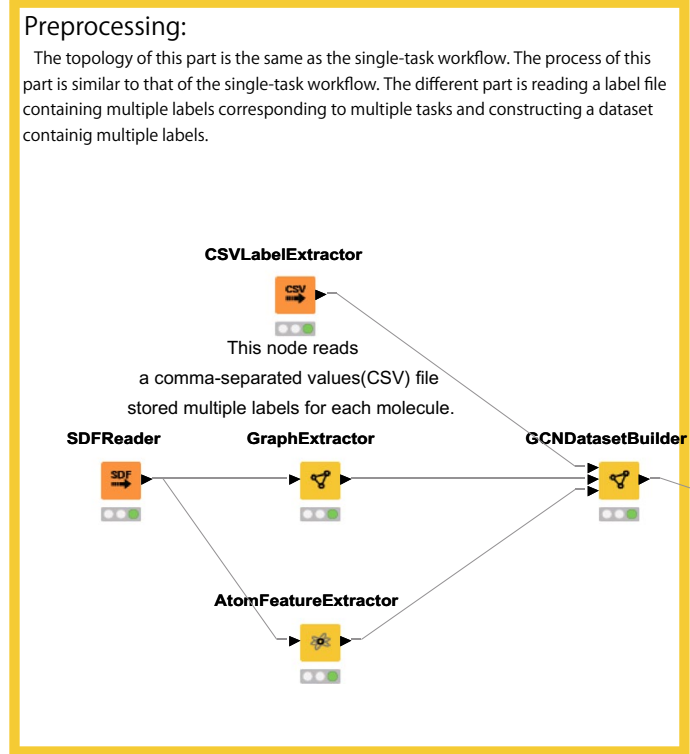

Training and evaluation:

This part carries out the hold out evaluation considering multiple tasks, i.e., the training a GCN model for the all tasks and evaluate the model using the test dataset for each task.

Fig. 5 Single-task workflow for the hold-out procedure using the KNIME interface (Upper). Multi-task workflow for the hold-out procedure (Lower)

\section{AddModality}

To change from single-task to multi-modal, AddModality node should be added next to the GCNDatasetBuilder node.

The visualization process shown at the bottom-right of Fig. 6 requires a specific computation time depending on the number of molecules to be visualized, as the computation time for the integrated gradient method for each molecule is $1-5 \mathrm{~s}$ during GPU execution. To reduce the size of the dataset, GCNDatasetSplitter can be used for selecting a part of the dataset.

\section{Python interface}

The kGCN system also provides a Python library for programmers to more precisely tune the setting of the analysis. The kGCN system can be used in a manner similar to any standard library and supports pip, a Python standard package manager. Furthermore, the kGCN system can be used in the Jupyter notebook, which is an interactive interface. Therefore, the users can easily explore this 


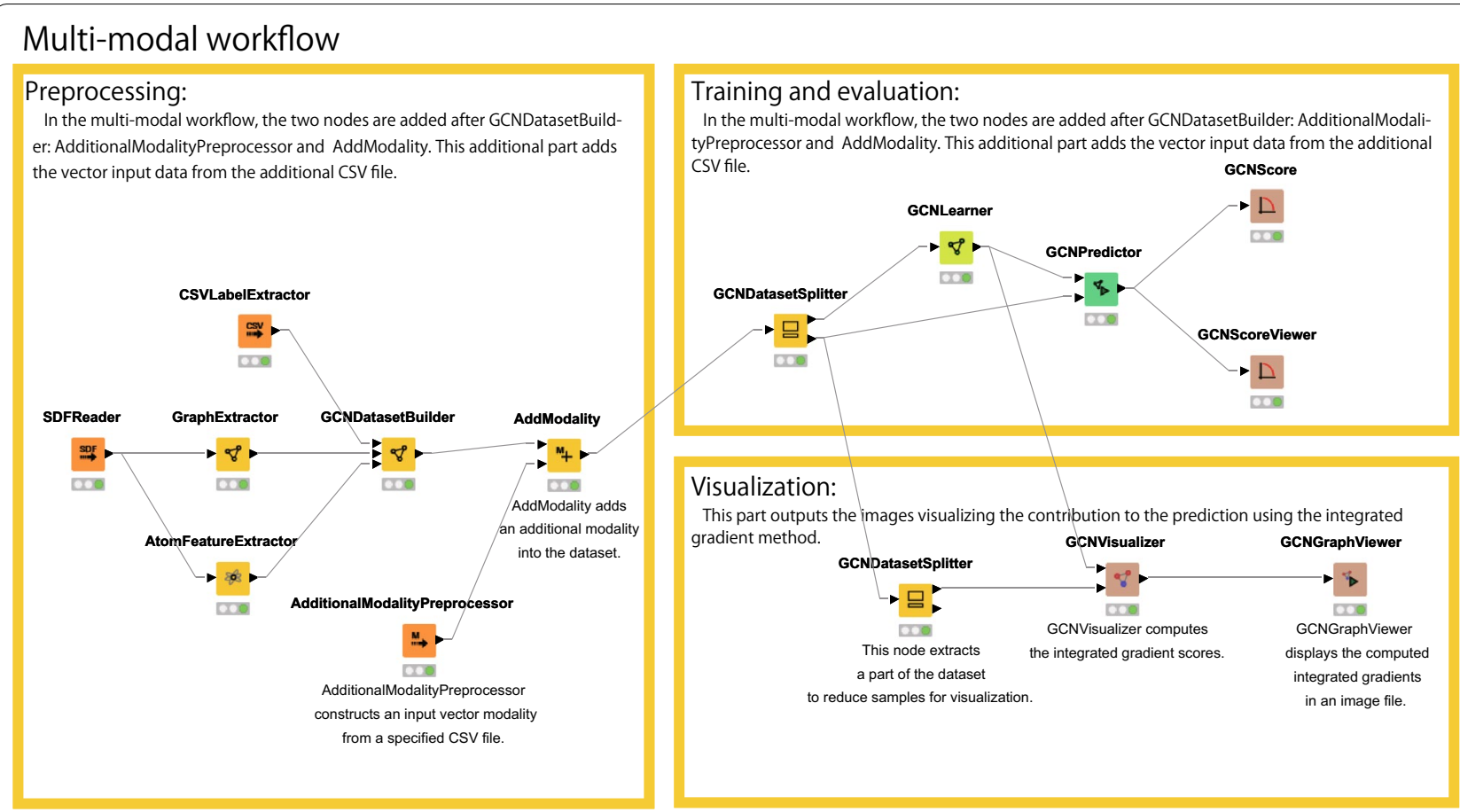

Fig. 6 Multi-modal workflow for the hold-out procedure

library using google collaboratory, a cloud environment for the execution of Python programs.

The kGCN system adopts an interface similar to scikitlearn, a defacto standard machine learning library in Python. Therefore, the process employing the kGCN library includes preprocessing, training by fit methods, and evaluation by pred method, in this order. The users can easily access the kGCN library in a similar manner to that of scikit-learn. Furthermore, designing a neural network, which is necessary for using kGCN, is easy if users are familiar with Keras because $\mathrm{kGCN}$ is compatible with the Keras library, and the users can easily design a neural network such as Keras.

To demonstrate a wide applicability of the present framework, three sample programs comprising the datasets and scripts using the standard functions of kGCN are available in the framework web pages. In addition to these examples, the application of kGCN for a reaction prediction has been reported in a prior study [36], where the visualized reaction centers predicted by GCNs were consistent with reaction centers reported in the literature. This literature report used GCNs for reaction prediction on the kGCN system.

\section{Flexible user interfaces}

As described in the introduction and implementation sections, kGCN provides KNIME GUI, a commandline interface, and a programming interface to support various types of users with various skill levels. For example, an easy-to-use high-layer GUI can assist the chemists with limited programming knowledge in using kGCN and understand SAR at a molecular level. Contrarily, for machine learning professionals with good programming skills, it is expected that they will focus on the improvement of algorithms using a low-layer python interface. By using a Python interface, the users can make machine learning procedures more flexible and incorporate the $\mathrm{kGCN}$ functions into the user specific programs such as web services. The users with good programming skills can also use the command-line interface to automate data-analysis procedures using the $\mathrm{kGCN}$ functions because it is easy to construct a pipeline combined with other commands such as Linux commands.

\section{Results}

For applications of $\mathrm{kGCN}$, this section describes the prediction of the assay results of a protein based on the molecular structure. The prediction of compoundprotein interactions (CPIs) has played an important role in drug discovery [38], and CPI prediction methods using deep learning have achieved excellent results $[4,14-16]$. In this study, the applicability of kGCN to CPI prediction is demonstrated as an example of single-task/multi-task/multi-modal GCNs. The singletask GCN predicts the activity against a protein based on the chemical structure represented as a graph. The 
Table 1 Number of compounds in our dataset

\begin{tabular}{lc}
\hline Assay type & \#Compounds \\
\hline MMP-3 & 2095 \\
MMP-9 & 2829 \\
MMP-12 & 533 \\
MMP-13 & 2607 \\
\hline
\end{tabular}

multi-task GCN predicts the activities against multiple proteins from a chemical structure. Although singletask and multi-task GCNs do not use the information related to proteins, multi-modal neural networks predict the activity from information of both the protein sequence and chemical structure.

For this examination, a dataset was prepared from the ChEMBL ver.20 database. The threshold for active/inactive was defined as $30 \mathrm{uM}$. This dataset consists of four types of matrix metalloprotease inhibition assays, MMP3, MMP-9, MMP-12, and MMP-13. The number of compounds for each assay are listed in Table 1. These MMPs were selected because relatively large amounts of data were available for these in the ChEMBL dataset [39].

$\mathrm{kGCN}$ provides many types of descriptors for a compound and protein. For example, kGCN allows graph representation for GCN and vector representation, such as ECFP [40] and DRAGON [41], for standard neural networks. Additionally, to represent a protein, kGCN uses an amino-acid sequence and vector representation such as PROFEAT descriptors [42]. This application uses graph representation for a compound and sequence representation for a sequence.

To simplify the experiment, the molecules with greater than 50 atoms were removed. As the dataset was unbalanced, negative data corresponding to inactivity were selected in the same manner [14]. Negative data was generated to equalize the number of negative and positive data for each assay.

Such preprocessing can be realized using the $\mathrm{kgcn}$ chem command included in the section describing the command-line interface.

Figure 7 shows the area under the curve in the receiver operator characteristic curve (ROC-AUC) of five-fold cross-validation. This result shows that the multi-modal approach outperforms the other approaches. The reason for a better ROC-AUC of the prediction with multi-modal approach is speculated to be the use of sequence-related information of the target proteins in addition to the graph representation of the compounds. This result is consistent with the reported results which indicate that the sequence descriptor contributes to improved accuracy $[4,14-16]$.

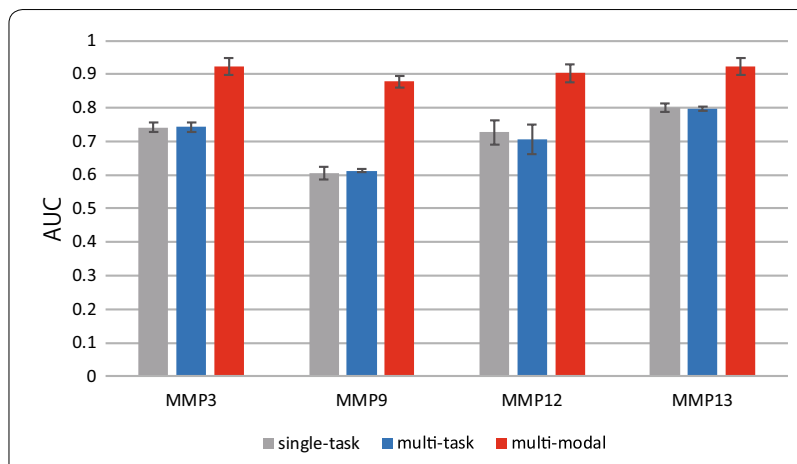

Fig. 7 AUCs obtained from five-fold cross-validation

a

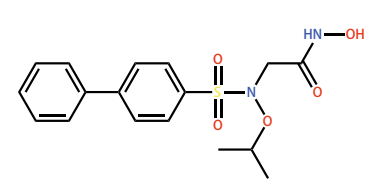

Fig. 8 a Chemical structure. b Atomic contributions to the predicted MMP-9 activity. Red color represents the positive contribution to the prediction (MMP-9 active in this case). Blue color represents the negative contribution (not active)

kGCN allows the visualization of the atomic contributions to the prediction result, as shown in Fig. 8b. The compound, N-hydroxy-2-[N-(propan-2-yloxy) [1,1'-biphenyl]-4-sulfonamido]acetamide (Fig. 8a), is used for this prediction and its reported activity $200 \mathrm{nM}$ (IC50) against MMP-9 [43]. The label of this compound for MMP-9 in the dataset is active, and the activity predicted for this compound in single-task mode is correct (probability of active label is 0.964). This compound possesses a hydroxamic acid group $(-\mathrm{C}(=\mathrm{O}) \mathrm{NHOH})$, and it is well-known that many MMP inhibitors have a hydroxamic group. The crystallographic structure of a complex of MMP-9 and this compound has been previously reported [44]. MMP-9 is a zinc protease, and the hydroxamic acid group of the above compound is coordinated to the zinc ion of MMP-9. The positive contributions of $\mathrm{OH}, \mathrm{NH}$, and carbonyl oxygen of the hydroxamic acid group shown in Fig. 8b are consistent with the interaction of the hydroxamic group with zinc of MMP-9.

Such visualization can be used to confirm the validity of the prediction by comparing the atomic contributions toward the prediction with structure-activity and/ or -property relationships. Additionally, this visualization can be useful for drug designing to improve the activity, physicochemical properties and/or ADMET 
properties by modifying the chemical moieties that contribute negatively to the prediction.

\section{Conclusion}

For assisting various users including chemists and cheminformaticians, an open-source GCN tool, kGCN, is described. To support the users with various levels of programming skills, kGCN provides three interfaces: a GUI using the KNIME platform for users with limited programming skills such as chemists, as well as command-line and Python library interfaces for the advanced users such as cheminformaticians and data scientists. Three steps including preprocessing, model tuning, and interpretation of results, required for building a prediction model and utilization of prediction results. kGCN supports these three steps by including functions such as the automatic preparation of graph representation based on the chemical structures for pre-processing, Bayesian optimization for automatic optimization of the hyper-parameters of the neural networks for model tuning, the integrated gradient method to visualize the atomic contribution toward the prediction result for interpretation. In terms of the approaches used for prediction, kGCN supports singletask, multi-task, and multi-modal predictions. The CPI prediction for four assays of matrixmetalloprotease inhibition, MMP-3, $-9,-12$, and -13 , is performed as a representative case study using $\mathrm{kGCN}$. Multi-modal prediction shows higher accuracy than those of the single-task and multi-task predictions. Additionally, the visualization of atomic contribution to the prediction indicated that hydroxamate group of the compound exhibits a positive contribution to the activity and this is consistent with the known structure-activity relationships. Such visualization is useful for the validation of the models and designing new molecules based on the model. This also allows the realization of "explainable AI" for understanding the factors influencing the AI prediction which are typically a black-box.

$\mathrm{kGCN}$ is available at https://github.com/clinfo/kGCN. Various examples such as Jupyter notebooks are also provided. Future works will include supporting new methods of graph neural networks because graph neural networks are a hot topic at present and new methods, e.g., graph attention and pooling, are being actively developed. We will proactively adopt these new methods and continue to develop kGCN so that various users can easily apply such latest methods to appropriately analyze the data in their hands and understand the reasons for the predictions. Also, we are going to gather the user feedback and improve kGCN for better usability.

\section{Authors' contributions}

RK; Designed and implemented the software, analysed data, and co-wrote the paper. Sl; Designed and implemented the software, analysed data, and co-wrote the paper. MO; analysed data and co-wrote the paper. $\mathrm{Hl}$; analysed data and co-wrote the paper. TH and $\mathrm{YO}$; supervised the research. All authors provided critical feedback and helped shape the research, analysis and manuscript. All authors read and approved the final manuscript.

\section{Funding}

This paper is based on a part of results obtained from a project commissioned by the New Energy and Industrial Technology Development Organization (NEDO).

\section{Availability of data and materials}

Project name: kGCN. Project home page: https://github.com/clinfo/kGCN. Operating system(s): Platform independent(Ubuntu 18.04, and CentOS 7 are mainly supported). Programming language: Python. Other requirements: python3 (> 3.6), tensorflow. License: https://github.com/clinfo/kGCN/blob/ master/LICENSE. Any restrictions to use by non-academics: licence needed.

\section{Competing interests}

The authors declare that they have no competing interests.

\section{Author details}

${ }^{1}$ Graduate School of Medicine, Kyoto University, Shogoin-kawaharacho, Sakyo-ku, Kyoto 606-8507, Japan. ${ }^{2}$ Graduate School of Pharmaceutical Sciences, Kyoto University, Yoshida, Sakyo-ku, Kyoto 606-8501, Japan. ${ }^{3}$ Medical Sciences Innovation Hub Program, RIKEN Cluster for Science, Technology and Innovation Hub, Tsurumi-ku, Kanagawa, Kanagawa 230-0045, Japan. ${ }^{4}$ RIKEN Center for Biosystems Dynamics Research, Tsurumi-ku, Kanagawa, Kanagawa 230-0045, Japan.

Received: 17 February 2020 Accepted: 28 April 2020

Published online: 12 May 2020

\section{References}

1. Gawehn E, Hiss JA, Schneider G (2016) Deep learning in drug discovery. Mol Inform 35(1):3-14. https://doi.org/10.1002/minf.201501008

2. Goh GB, Hodas NO, Vishnu A (2017) Deep learning for computational chemistry. J Comput Chem 38(16):1291-1307

3. Elton DC, Boukouvalas Z, Fuge MD, Chung PW (2019) Deep learning for molecular design - a review of the state of the art. Mol Syst Design Eng 4(4):828-849

4. Torng W, Altman RB (2019) Graph convolutional neural networks for predicting drug-target interactions. J Chem Inform Model 59(10):4131-4149

5. Ma J, Sheridan RP, Liaw A, Dahl GE, Svetnik V (2015) Deep neural nets as a method for quantitative structure-activity relationships. J Chem Inform Model 55(2):263-274

6. Schneckener S, Grimbs S, Hey J, Menz S, Osmers M, Schaper S, Hillisch A, Göller AH (2019) Prediction of oral bioavailability in rats: transferring insights from in vitro correlations to (deep) machine learning models using in silico model outputs and chemical structure parameters. J Chem Inform Model 59(11):4893-4905

7. Wegner JK, Sterling A, Guha R, Bender A, Faulon J-L, Hastings J, O'Boyle $\mathrm{N}$, Overington J, Van Vlijmen H, Willighagen E (2012) Cheminformatics. Commun ACM 55(11):65-75

8. Kearnes S, McCloskey K, Berndl M, Pande V, Riley P (2016) Molecular graph convolutions: moving beyond fingerprints. J Comput Aided Mol Des 30(8):595-608

9. Gilmer J, Schoenholz SS, Riley PF, Vinyals O, Dahl GE (2017) Neural message passing for quantum chemistry. In: Proceedings of the 34th International Conference on Machine Learning, vol. 70, pp 1263-1272

10. Duvenaud DK, Maclaurin D, Iparraguirre J, Bombarell R, Hirzel T, AspuruGuzik A, Adams RP (2015) Convolutional networks on graphs for learning molecular fingerprints. Adv Neural Inform Process Syst 28:2224-2232

11. Jin W, Coley CW, Barzilay R, Jaakkola T (2017) Predicting organic reaction outcomes with weisfeiler-lehman network. In: Proceedings of the 31st International Conference on Neural Information Processing Systems, pp 2604-2613 
12. Berthold MR, Cebron N, Dill F, Gabriel TR, Kotter T, Mein IT, Ohl P, Thiel K, Wiswedel B (2009) Knime - the konstanz information miner: version 20 and beyond. ACM SIGKDD Explorat Newslett 11(1):26-31

13. Goodfellow I, Bengio Y, Courville A (2016) Deep Learning. MIT Press

14. Hamanaka M, Taneishi K, Iwata H, Ye J, Pei J, Hou J, Okuno Y (2017) Cgbvs-dnn: prediction of compound-protein interactions based on deep learning. Mol Inform 36(1-2):1600045

15. Nguyen TT, Nguyen T, Le DH, Quinn H, Venkatesh S (2020) Predicting drug-target binding affinity with graph neural networks. bioRxiv. https ://doi.org/10.1101/684662. https://www.biorxiv.org/content/early /2020/01/22/684662.full.pdf

16. Tsubaki M, Tomii K, Sese J (2019) Compound-protein interaction prediction with end-to-end learning of neural networks for graphs and sequences. Bioinformatics 35(2):309-318

17. Ramsundar B, Liu B, Wu Z, Verras A, Tudor M, Sheridan RP, Pande V (2017) Is multitask deep learning practical for pharma? J Chem Inform Model 57(8):2068-2076

18. Sanyal S, Balachandran J, Yadati N, Kumar A, Rajagopalan P, Sanyal S, Talukdar P (2018) MT-CGCNN: Integrating crystal graph convolutional neural network with multitask learning for material property prediction. arXiv preprint arXiv:1811.05660

19. Liu K, Sun X, Jia L, Ma J, Xing H, Wu J, Gao H, Sun Y, Boulnois F, Fan J (2019) Chemi-net: a molecular graph convolutional network for accurate drug property prediction. Int J Mol Sci 20(14):3389

20. Selvaraju RR, Cogswell M, Das Vedantam AR, Parikh D, Batra D (2017) Grad-cam: visual explanations from deep networks via gradient-based localization. In: Proceedings of the IEEE International Conference on Computer Vision, pp 618-626

21. Smilkov D, Thorat N, Kim B, Viegas F, Wattenberg M (2017) Smoothgrad: removing noise by adding noise. arXiv preprint arXiv:1706.03825

22. Sundararajan M, Taly A, Yan Q (2017) Axiomatic attribution for deep networks. In: Proceedings of the 34th International Conference on Machine Learning, vol 70, pp 3319-3328. JMLR.org

23. Snoek J, Larochelle H, Adams RP (2012) Practical bayesian optimization of machine learning algorithms. In: Proceedings of the 25th International Conference on Neural Information Processing Systems, vol 2, pp 2951-2959

24. Abadi M, Barham P, Chen J, Chen Z, Davis A, Dean J, Devin M, Ghemawat S, Irving G, Isard M, et al. (2016) Tensorflow: a system for large-scale machine learning. In: 12th USENIX Symposium on Operating Systems Design and Implementation, pp 265-283

25. Ramsundar B, Eastman P. Walters P, Pande V (2019) Deep learning for the life sciences. O'Reilly Media inc.,

26. pfnet research: chainer-chemistry. https://github.com/pfnet-research/ chainer-chemistry

27. Popova M Openchem: deep learning toolkit for computational chemistry and drug design. https://github.com/Mariewelt/OpenChem

28. Tokui S, Oono K, Hido S, Clayton J Chainer (2015) A next-generation open source framework for deep learning. In: Proceedings of Workshop on Machine Learning Systems (LearningSys) in the Twenty-ninth Annual Conference on Neural Information Processing Systems (NIPS), vol 5, pp $1-6$

29. Landrum G (2018) RDKit: open-source cheminformatics. http://www.rdkit .org (Accessed August 21 2019)
30. Kipf TN, Welling M (2017) Semi-supervised classification with graph convolutional networks. In: International Conference on Learning Representations

31. Altae-Tran H, Ramsundar B, Pappu AS, Pande V (2017) Low data drug discovery with one-shot learning. ACS Cent Sci 3:283-293

32. Ioffe S, Szegedy C (2015) Batch normalization: accelerating deep network training by reducing internal covariate shift. arXiv preprint. arXiv:1502.03167

33. Montanari F, Kuhnke L, Laak A Ter, Clevert D-A (2020) Modeling physicochemical admet endpoints with multitask graph convolutional networks. Molecules 25(1):44

34. Abadi M, Agarwal A, Barham P, Brevdo E, Chen Z, Citro C, Corrado GS, Davis A, Dean J, Zheng X (2015) TensorFlow: large-scale machine learning on heterogeneous systems. https://www.tensorflow.org/ (Accessed 21 August 2019)

35. Chollet F, et al (2015) Keras. https://github.com/fchollet/keras

36. Ishida S, Terayama K, Kojima R, Takasu K, Okuno Y (2019) Prediction and interpretable visualization of retrosynthetic reactions using graph convolutional networks. J Chem Inform Model 59(12):5026-5033

37. The GPyOpt authors: GPyOpt (2016) A bayesian optimization framework in Python. http://github.com/SheffieldML/GPyOpt

38. Keiser MJ, Setola V, Irwin JJ, Laggner C, Abbas Al, Hufeisen SJ, Jensen NH, Kuijer MB, Matos RC, Tran TB et al (2009) Predicting new molecular targets for known drugs. Nature 462(7270):175-181

39. Gimeno A, Beltrán-Debón R, Mulero M, Pujadas G, Garcia-Vallvé S (2020) Understanding the variability of the $S 1$ 'pocket to improve matrix metalloproteinase inhibitor selectivity profiles. Drug Discov Today 25(1):38-57

40. Rogers D, Hahn M (2010) Extended-connectivity fingerprints. J Chem Informat Model 50(5):742-754

41. Mauri A, Consonni V, Pavan M, Todeschini R (2006) Dragon software: an easy approach to molecular descriptor calculations. MATCH Commun Math Comput Chem 56(2):237-248

42. Zhang P, Tao L, Zeng X, Qin C, Chen S, Zhu F, Li Z, Jiang Y, Chen W, Chen Y-Z (2016) A protein network descriptor server and its use in studying protein, disease, metabolic and drug targeted networks. Brief Bioinform 18(6):1057-1070

43. Rossello A, Nuti E, Carelli P, Orlandini E, Macchia M, Nencetti S, Zandomeneghi M, Balzano F, Barretta GU, Albini A, Benelli R, Cercignani G, Murphy G, Balsamo A (2005) Ni-propoxy-n-biphenylsulfonylaminobutylhydroxamic acids as potent and selective inhibitors of $\mathrm{mmp}-2$ and mt1-mmp. Bioorg Med Chem Lett 15(5):1321-1326

44. Antoni C, Vera L, Devel L, Catalani MP, Czarny B, Cassar-Lajeunesse E, Nuti E, Rossello A, Dive V, Stura EA (2013) Crystallization of bi-functional ligand protein complexes. J Struct Biol 182(3):246-254

\section{Publisher's Note}

Springer Nature remains neutral with regard to jurisdictional claims in published maps and institutional affiliations.

Ready to submit your research? Choose BMC and benefit from

- fast, convenient online submission

- thorough peer review by experienced researchers in your field

- rapid publication on acceptance

- support for research data, including large and complex data types

- gold Open Access which fosters wider collaboration and increased citations

- maximum visibility for your research: over $100 \mathrm{M}$ website views per year

At BMC, research is always in progress.

Learn more biomedcentral.com/submissions 